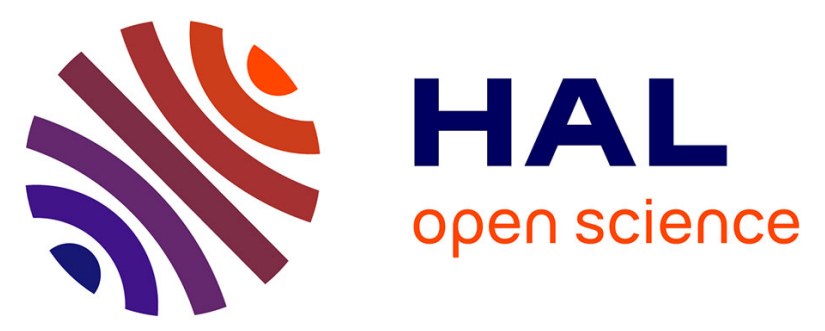

\title{
No difference in HIV-1 integrase resistance between CSF and blood compartments Short title: HIV-1 integrase resistance in compartments
}

Basma Abdi, Mouna Chebbi, Marc Wirden, Elisa Teyssou, Sophie Sayon, Romain Palich, Sophie Seang, Marc-Antoine Valantin, Anne Simon, Roland Tubiana, et al.

\section{To cite this version:}

Basma Abdi, Mouna Chebbi, Marc Wirden, Elisa Teyssou, Sophie Sayon, et al.. No difference in HIV1 integrase resistance between CSF and blood compartments Short title: HIV-1 integrase resistance in compartments. Journal of Antimicrobial Chemotherapy, 2021, 10.1093/jac/dkab064 . hal-03174456

\section{HAL Id: hal-03174456 https://hal.sorbonne-universite.fr/hal-03174456}

Submitted on 19 Mar 2021

HAL is a multi-disciplinary open access archive for the deposit and dissemination of scientific research documents, whether they are published or not. The documents may come from teaching and research institutions in France or abroad, or from public or private research centers.
L'archive ouverte pluridisciplinaire HAL, est destinée au dépôt et à la diffusion de documents scientifiques de niveau recherche, publiés ou non, émanant des établissements d'enseignement et de recherche français ou étrangers, des laboratoires publics ou privés. 
1 No difference in HIV-1 integrase resistance between CSF and blood compartments

2 Short title: HIV-1 integrase resistance in compartments

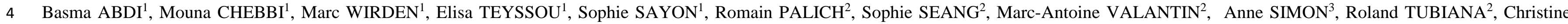

5 KATLAMA $^{2}$, Vincent CALVEZ ${ }^{1}$, Anne-Geneviève MARCELIN ${ }^{1}$, Cathia SOULIE ${ }^{1 *}$,

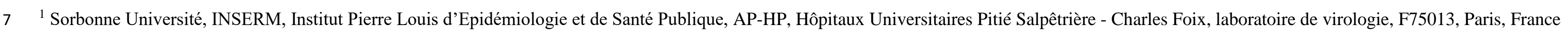

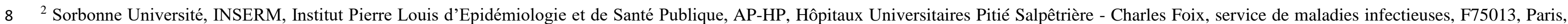
9 France

$10{ }^{3}$ AP-HP, Hôpitaux Universitaires Pitié Salpêtrière - Service de médecine interne, F75013, Paris, France

\section{CORRESPONDING AUTHOR}

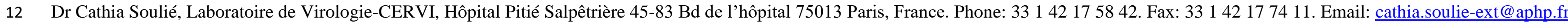




\section{ABSTRACT}

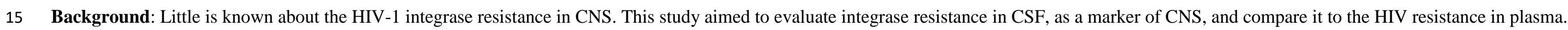

16 Methods: The HIV integrase was sequenced both in plasma and CSF for 59 HIV-1 patients. The clinical and biological data were collected from clinical routine care.

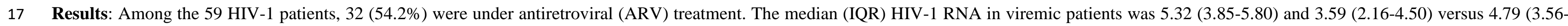

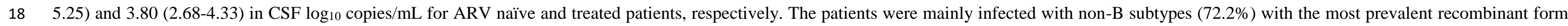

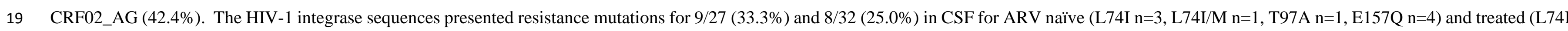

$20 \mathrm{n}=6, \mathrm{~L} 74 \mathrm{M} \mathrm{n}=1,1 \mathrm{~T} 97 \mathrm{~A} \mathrm{n}=1,1 \mathrm{~N} 155 \mathrm{H} \mathrm{n}=1$ ) patients, respectively. Integrase resistance mutations in CSF were similar to that in plasma, except for $1 / 59$ patients.

21 Conclusions: This work shows similar integrase resistance profiles in CNS and plasma in a population of HIV-1 viremic patients. 


\section{INTRODUCTION}

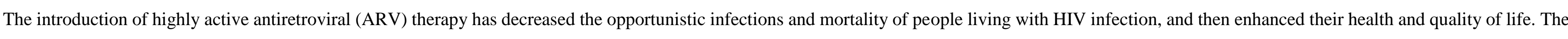

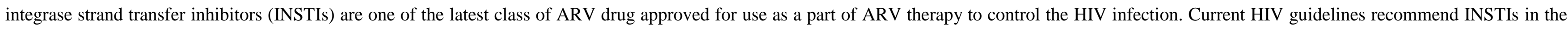

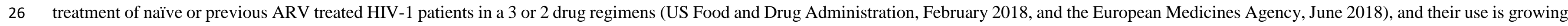
worldwide.

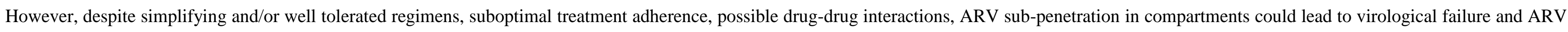

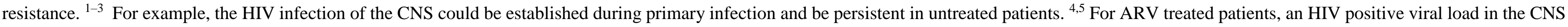

\section{METHODS}

\section{Patients}




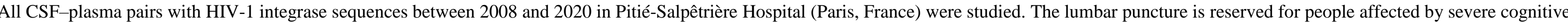

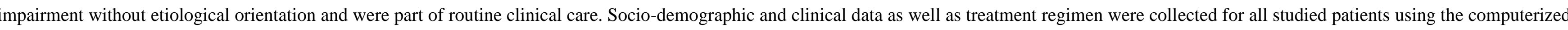

38 medical chart NADIS ${ }^{\circledR}$ for which all patients provided signed consent.

\section{Genotyping resistance testing}

41 The integrase resistance mutations were determined using the ANRS consensus technique

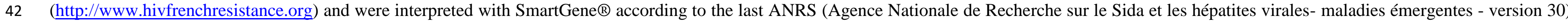

43 or Stanford HIVDB 8.9.1 algorithm

\section{Phylogenetic analysis}

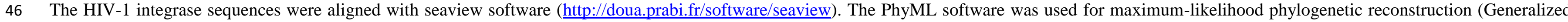

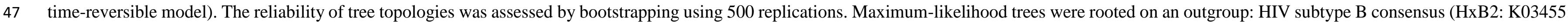
48 www.hiv.lanl.gov). Tree figures were viewed and modified with FigTree software (http://tree.bio.ed.ac.uk/software/figtree/). 


\section{Statistical methods}

Quantitative variables were summarized by medians and IQR and qualitative variables by percent

\section{RESULTS}

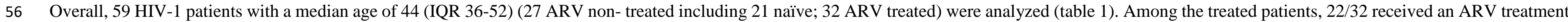

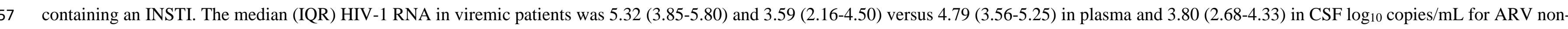

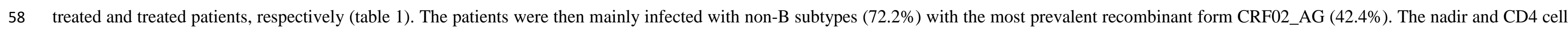
59 counts were in median (IQR) 46 (16-20) and 113 (29-274) cells/mm³ , respectively (table 1).

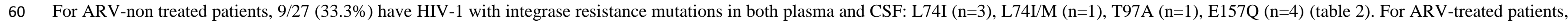

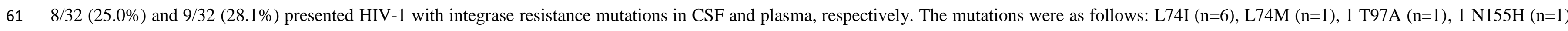

62 both in plasma and CSF. Only 1 E138K, associated to L74I, was present only in plasma for one patient (table 2). 


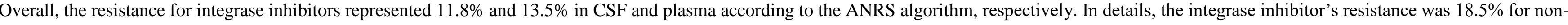

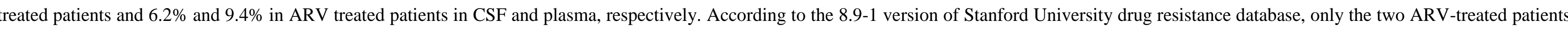
with HIV-1 major integrase inhibitor resistance mutations were considered resistant to INSTI, in plasma (E138K) and both in CSF and plasma (N155H).

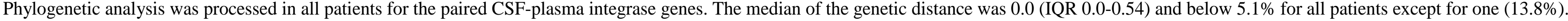

\section{DISCUSSION}

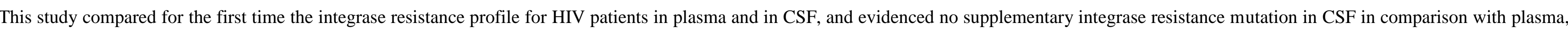

except for 1/59 patients.

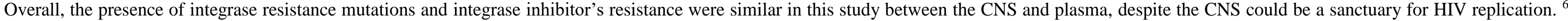

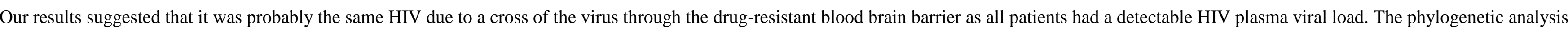

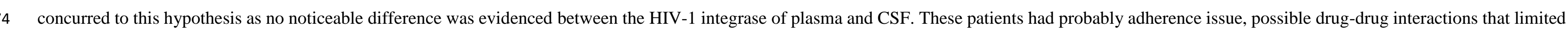

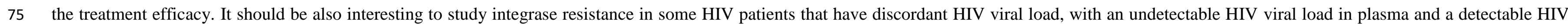




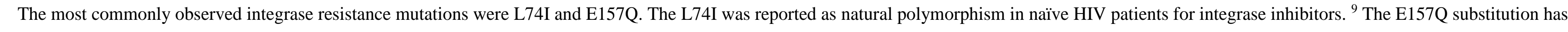

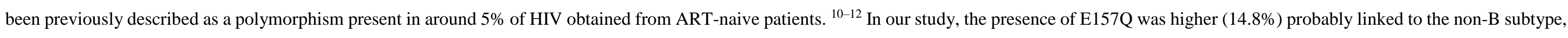

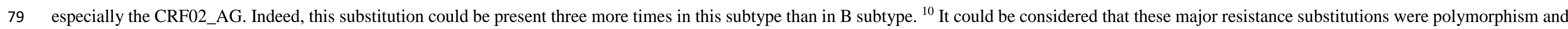
80 not acquired resistance mutations.

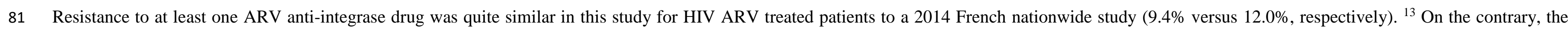

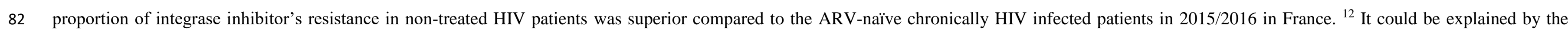

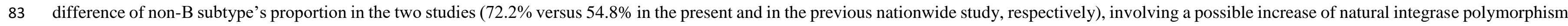

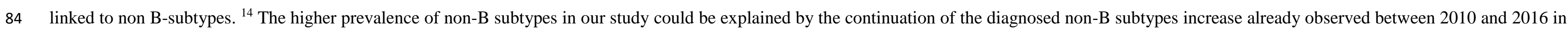

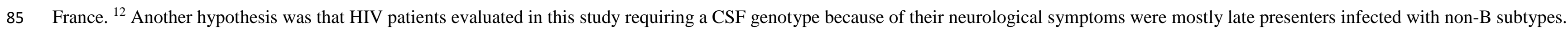

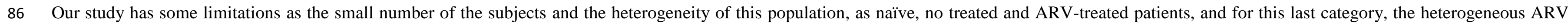

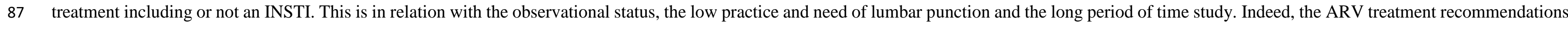

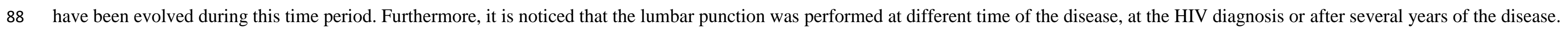


ACKNOWLEDGMENT

This work was supported by the Agence Nationale de Recherche sur le Sida et les hépatites virales - maladies émergentes (ANRS).

111

\section{FUNDING}

113 This study was carried out as a part of our routine work.

\section{TRANSPARENCY DECLARATION}

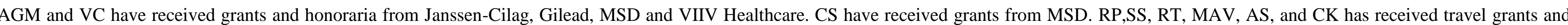

117 advisory fees from Gilead, ViiV Healthcare and Merck. All other authors: none to declare. 


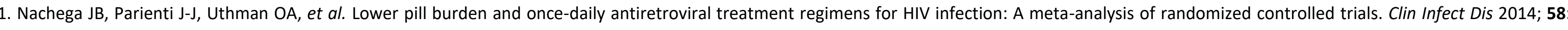
$1297-307$.

123 2. Bangsberg DR, Perry S, Charlebois ED, et al. Non-adherence to highly active antiretroviral therapy predicts progression to AIDS. AIDS 2001; 15: 1181-3.

3. Gardner EM, Sharma S, Peng G, et al. Differential adherence to combination antiretroviral therapy is associated with virological failure with resistance. AIDS 2008; 22: 75-82.

4. Spudich SS, Huang W, Nilsson AC, et al. HIV-1 chemokine coreceptor utilization in paired cerebrospinal fluid and plasma samples: a survey of subjects with viremia. J Infect Dis 2005; 191: 890-8.

5. Pilcher CD, Shugars DC, Fiscus SA, et al. HIV in body fluids during primary HIV infection: implications for pathogenesis, treatment and public health. AIDS 2001; 15: 837-45.

6. Anderson AM, Muñoz-Moreno JA, McClernon DR, et al. Prevalence and Correlates of Persistent HIV-1 RNA in Cerebrospinal Fluid During Antiretroviral Therapy. J Infect Dis 2017; 215: 105-13.

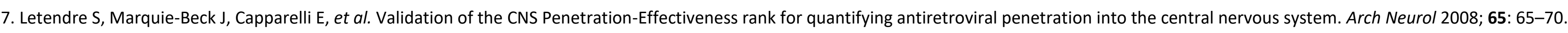

9. Lataillade M, Chiarella J, Kozal MJ. Natural polymorphism of the HIV-1 integrase gene and mutations associated with integrase inhibitor resistance. Antivir Ther (Lond) 2007; 12: 563-70.

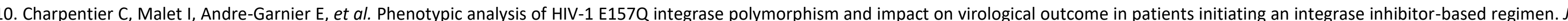
Antimicrob Chemother 2018; 73: 1039-44.

133 11. Saladini F, Giannini A, Boccuto A, et al. The HIV-1 integrase E157Q polymorphism per se does not alter susceptibility to raltegravir and dolutegravir in vitro. AIDS 2017; 31: 2307-9.

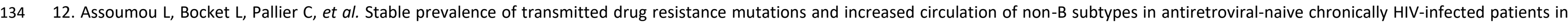
135 2015/2016 in France. J Antimicrob Chemother 2019;74(5):1417-1424.

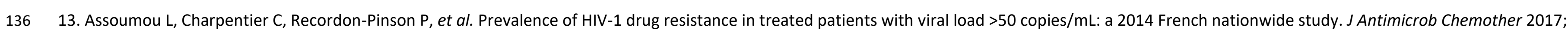
137 72: 1769-73. 
Age, years median (IQR)

$44(36-52)$

$42(35-48)$

$48(37-53)$

Male, $\mathbf{n}(\%)$

$35(59.3)$

$15(55.5)$

$20(62.5)$

B subtype, $\mathbf{n}(\%)$

$17(28.8)$

$9(33.3)$

$8(25.0)$

Time since HIV diagnosis, months median (IQR)

$96.4(0.0-187.5)$

$1(0.3-145.1)$

$122.0(40.2-212.6)$

CSF HIV-1 RNA, $\log _{10}$ copies/mL median (IQR)

$4.2(3.13-4.95)$

$4.79(3.56-5.25)$

$3.80(2.68-4.33)$

Plasma HIV-1 RNA, $\log _{10}$ copies/mL median (IQR)

$4.71(3.14-5.44)$

$5.32(3.85-5.80)$

$3.59(2.16-4.50)$

Plasma zenith HIV-1 RNA, $\log _{10}$ copies/mL median (IQR) 
Total time under ARV treatment, weeks median (IQR)

Current treatment, $\%$

\begin{tabular}{|c|c|c|}
\hline NRTIs+Pis & $6(10.2)$ & $6(18.7)$ \\
\hline NRTIs+PIs+MVC & $3(5.1)$ & $3(9.4)$ \\
\hline NRTIs+INIs & $16(27.1)$ & $16(50.0)$ \\
\hline PIs + INIs & $3(5.1)$ & $3(9.4)$ \\
\hline NRTIs + Pis + INIs & $2(3.4)$ & $2(6.3)$ \\
\hline Pis & $1(1.7)$ & $1(3.1)$ \\
\hline NRTIs + NNRTIs + INIs & $1(1.7)$ & $1(3.1)$ \\
\hline
\end{tabular}


*6 patients of the ARV non-treated group at time of the HIV genotypes have received ARV treatment in the past. 


\begin{tabular}{|c|c|c|c|c|c|c|c|c|c|c|c|c|c|c|c|}
\hline Patient & Age & Sex & $\begin{array}{c}\text { Time since } \\
\text { HIV } \\
\text { diagnosis }\end{array}$ & $\begin{array}{l}\text { Total time under } \\
\text { ARV treatment } \\
\text { (duration of the } \\
\text { cessation of } \\
\text { treatment) }\end{array}$ & $\begin{array}{l}\text { Time under } \\
\text { current ARV } \\
\text { treatment }\end{array}$ & $\begin{array}{c}\text { Integrase } \\
\text { resistance } \\
\text { mutation } \\
\text { CSF }\end{array}$ & $\begin{array}{l}\text { Integrase resistance } \\
\text { mutation } \\
\text { Plasma }\end{array}$ & CSF HIV VL & Plasma HIV VL & Zenith HIV VL & Nadir & CD4+ & CD8+ & Subtype & ARV \\
\hline 1 & 31 & $\mathrm{~F}$ & 0 & & & L74I/M & L74I/M & 5.3 & 6.0 & 6.0 & 46 & 79 & 664 & CRF02_AG & naive \\
\hline 2 & 49 & $\mathrm{~F}$ & 970 & $17(326)$ & & L74I & L74I & 4.7 & 2.5 & 6.8 & 31 & 99 & 812 & A & no \\
\hline 3 & 42 & M & 379 & & & L74I & L74I & 4.8 & 5.6 & 5.3 & 4 & 20 & 142 & CFR02_AG & naive \\
\hline 4 & 24 & $\mathrm{~F}$ & 350 & & & L74I & L74I & 6.3 & 5.9 & 6.1 & 10 & 24 & 244 & A & naive \\
\hline 5 & 54 & M & 2 & & & T97A & T97A & 4.4 & 3.7 & 3.7 & 46 & 46 & 692 & B & naive \\
\hline 6 & 47 & M & 820 & & & E157Q & E157Q & 3.4 & 5.2 & 5.6 & 9 & 9 & 285 & B & naive \\
\hline 7 & 33 & $\mathrm{~F}$ & 791 & $667(116)$ & & E157Q & E157Q & 4.8 & 4.7 & 5.9 & 263 & 334 & 1424 & CFR02_AG & no \\
\hline 8 & 25 & $\mathrm{~F}$ & 4 & & & E157Q & E157Q & 5.1 & 5.4 & 5.4 & 205 & 205 & 228 & CFRO2_AG & naive \\
\hline 9 & 45 & $\mathrm{~F}$ & 1 & & & E157Q & E157Q & 3.9 & 5.1 & 5.0 & nd & nd & nd & CR02_AG & naive \\
\hline
\end{tabular}




\begin{tabular}{|c|c|c|c|c|c|c|c|c|c|c|c|c|c|c|c|}
\hline 10 & 54 & M & 384 & 226 & 46 & L74I & L74I & 4.5 & 3.6 & 5.0 & 16 & 195 & 1104 & CRF02_AG & $R A L+R T V+D R V+3 T C$ \\
\hline 11 & 52 & $\mathrm{~F}$ & 1263 & 1055 & 285 & L74I & L74I & 5.3 & 5.8 & 5.8 & 122 & nd & nd & A & $F T C+T D F+L P V+R T V$ \\
\hline 12 & 73 & M & 1137 & 1059 & 2 & L74I & L74I & 2.6 & 4.0 & 6.6 & 25 & 64 & 813 & CRF02_AG & $R T V+D R V+F T C+T D F$ \\
\hline 13 & 42 & M & 420 & 420 & 62 & L74I & L74I & 2.7 & 1.7 & 5.4 & 10 & 209 & 407 & CRF011_cpx & $R T V+A T V+D T G$ \\
\hline 14 & 49 & M & 485 & 485 & 15 & L74I & L74I & 2.8 & 2.6 & 2.8 & 174 & 174 & 641 & CRF02_AG & $L P V+R T V+3 T C+Z D V$ \\
\hline 15 & 58 & $M$ & 17 & 14 & 8 & L74I & L74I, E138K & 2.4 & 3.1 & 5.9 & 391 & 628 & 466 & A & $D T G+A B C+3 T C$ \\
\hline 16 & 39 & $\mathrm{~F}$ & 625 & 601 & 9 & L74M & L74M & 2.1 & 1.9 & 6.1 & 237 & 919 & 1081 & CRF02_AG & $R T V+T D F+F T C+A T V$ \\
\hline 17 & 52 & M & 381 & 202 & 70 & T97A & T97A & 4.2 & 5.5 & 6.3 & 7 & 14 & 754 & B & $R A L+A B C+3 T C+M V C$ \\
\hline 18 & 36 & $\mathrm{~F}$ & 577 & 577 & 50 & $\mathrm{~N} 155 \mathrm{H}$ & $\mathrm{N} 155 \mathrm{H}$ & 4.5 & 6.1 & 6.1 & 39 & 39 & 347 & CRF02_AG & RAL+RTV+ATZ \\
\hline
\end{tabular}

147 Table 2 : Characteristics of HIV patients with HIV integrase resistance mutations.

148 VL : Viral Load; nd: not done 
Time since diagnosis, total time under ARV treatment and Time under current ARV treatment are expressed in weeks. The HIV viral loads were expressed as log 10 copies/mL; The CD4. CD8 and nadir were expressed as cells $/ \mathrm{mm}^{3}$ 